\title{
Fatty Acid Requirements of Human Cutaneous Lipophilic Corynebacteria
}

\author{
By R. F. S M ITH \\ Department of Microbiology, Temple University School of Pharmacy, \\ Philadelphia, Pennsylvania I9140, U.S.A.
}

(Accepted for publication 6 November 1969)

\begin{abstract}
SUMMAR Y
Thirty-nine human cutaneous and eight bovine obligate-lipophilic corynebacteria were examined for their fatty acid requirements for growth. Twentyfive saturated and five unsaturated compounds did not support growth. Oleic acid in cis, trans and iso forms supported various degrees of growth. Erucic acid was also utilized but not ricinoleic and nervonic acids. Linoleic acid supported the growth of some strains but linolenic acid did not. These acids were not inhibitory to most strains tested. Three short-chain acids (acetic, lactic, propionic) and glycerol did not potentiate growth of the strains in the presence of oleic acid. In oleic acid broth, palmitic acid which did not support growth alone, stimulated I4 strains and inhibited three others. Strains of Corynebacterium bovis differed from the human strains in their growth response.
\end{abstract}

\section{INTRODUCTION}

Normal human skin supports an abundance of lipophilic corynebacteria (Marples, I969). These organisms require Tween 80 or oleic acid for growth in vitro (Pollock, Wainwright \& Mansion, 1949). Tween 80 is known to contain from 0.4 to $0.8 \%$ unesterified fatty acids including myristic, myristoleic, palmitic, palmitoleic and oleic acids (Stinson \& Solotorovsky, 1969); other Tween compounds also contain mixtures of fatty acids (Weary, I968). Except for general growth support, Tweens cannot be used to determine accurately the specific fatty acid requirements of bacteria. The cutaneous corynebacteria have not been previously examined for the spectrum of their fatty acid requirements with the exception of oleic acid; the results of such a study are reported here.

\section{METHODS}

Organisms. Obligate-lipophilic corynebacteria (LC) from human skin were isolated and classified by the methods of Smith $(1969 a, b)$. The maintenance and sources of reference stains were reported previously (Smith, I969a). Strains of Corynebacterium bovis were used in some experiments with the human strains.

Compounds. Lipids and other chemicals were obtained from the Nutritional Biochemical Corp., Cleveland, Ohio and Sigma Chemical Co., St Louis, Missouri, U.S.A. All compounds were $99 \%$ pure except for vaccenic, erucic and elaidic acids and soybean lecithin which were $95 \%$ pure. Water-soluble compounds were prepared in aqueous solutions and sterilized by filtration. Water-insoluble compounds were dissolved in $95 \%(\mathrm{v} / \mathrm{v})$ ethanol in water. All stock compounds were stored at $-20^{\circ}$. 
Basal medium and supplements. Nutrient broth (Difco) was prepared in concentrated form $(24 \mathrm{~g} . / 100 \mathrm{ml}$.) and washed 3 times with light petroleum. This lipid-deficient concentrate was adjusted so that $30 \mathrm{ml}$. was diluted to yield I 1 . of broth. Three lots of concentrate were prepared. Each lot made 91 . broth at final $\mathrm{pH} 7 \cdot 0$, obtained without adjustment. Previous experiments showed that human LC strains were incapable of growing in this medium unless supplemented with certain lipids. Growth response of non-lipophilic organisms was identical in each of the three test lots. Compounds were added to the autoclaved basal medium to give final concentrations of $10^{-4} \mathrm{M}$. Basal medium $+0 \cdot 1 \%$ Tween 80 was used as a growth control. Broth was dispensed aseptically in $5 \mathrm{ml}$. amounts.

Growth measurements. Cultures were grown on nutrient agar slopes (Difco) containing $0.05 \%$ Tween 80 for $48 \mathrm{hr}$. The bacteria were washed from the slopes with sterile 0.1 M-phosphate buffer ( $\mathrm{pH} \mathrm{7.4}^{2}$ containing $0.05 \%$ Triton X 100. Cell suspensions were adjusted to an extraction reading of 0.5 with a Bausch and Lomb Spectronic20 colorimeter $(650 \mathrm{~nm}$.) and samples of $0.05 \mathrm{ml}$., delivered from serological pipettes, were used to inoculate the tubes of medium. Using conventional plating methods on to agar (Smith, 1969 $b$ ) the inoculum was shown to contain about 100,000 viable bacteria. Cultures were incubated at $35^{\circ}$ for 7 days with intermittent shaking by hand. Growth response was considered positive when cultures reached a turbidity of $50 \%$ transmittance or less (a minimum of $\mathrm{I} \times 10^{7}$ bacteria $/ \mathrm{ml}$.).

\section{RESULTS}

Thirty-nine human cutaneous and eight bovine lipophilic corynebacterial (LC) strains were examined for ability to utilize and initiate growth on 38 different fatty acids and related compounds. Twenty-five saturated and five unsaturated compounds did not support the growth of any of the human or bovine strains (Table I). Seven unsaturated fatty acids and lecithin supported growth of the 39 human LC strains in varying degrees (Table 2). Elaidic (trans oleic) and cis oleic acids were the most effective compounds, followed by vaccenic (iso oleic) and erucic acids. Several strains grew equally well on more than one of the active compounds. With increased desaturation of the cis oleic acids (linoleic, linolenic), decreased growth response resulted. Six of eight Corynebacterium bovis strains did not grow with any of the seven unsaturated fatty acids or lecithin. Strains NIRD 66 and 126 were the only C. bovis strains that grew substantially in the presence of four of the seven unsaturated fatty acids (Table 2 ). One strain each of C. striatum, C. minutissimum, C. xerosis, C. diphtheriae, Brevibacterium linens, Enterobacter aerogenes and 3 strains of Staphylococcus epidermidis grew in the basal lipid-deficient broth and in the media containing each of the 38 individually tested compounds. There were no appreciable stimulatory or inhibitory effects by any of the compounds on these cultures. Basal medium + Tween 80 was generally adequate for growth.

Experiments were made to determine whether linoleic, linolenic and ricinoleic acids were inhibitory rather than not utilized. Each of these fatty acids was separately added to basal medium containing Tween 80 or oleic acid; Tween 80 and oleic acid media without supplement were used as controls for comparison (Table 3). Six strains were completely inhibited by linoleic acid and five strains by linolenic acid. Ricinoleic acid was not inhibitory. Sixteen strains grew in oleate broth containing separate 
supplements of $\mathrm{IO}^{-3} \mathrm{M}$ lactic, acetic or propionic acids and glycerol. None of these short-chain compounds potentiated growth of the 16 strains in oleic acid broth. Finally, 37 of 39 human LC strains were grown in broth containing $10^{-4} \mathrm{M}$ oleic and palmitic acids, and compared with growth in broth containing only oleic acid. Fourteen strains were stimulated in oleic broth by the addition of palmitic acid and three strains were inhibited.

Table I. Fatty acids and other compounds not supporting growth of human and bovine lipophilic corynebacteria

Compounds tested at $10^{-4} \mathrm{M}$ in basal lipid-free medium.

Saturated compounds:

Ethanol, n-butanol, ethylacetate, glycerol, monoacetin, triacetin, testosterone propionate

Acids:

acetic $\left(C_{1}\right)$, proprionic $\left(C_{2}\right), n$-butyric (etc), valeric, caproic, caprylic, capric, lauric, myristic, palmitic, margaric, stearic, arachidic, nonadecanoic, behenic, pyruvic.

Sebacic. Lactic acid. Poly- $\beta$-hydroxyl butyric

Unsaturated acids: Crotonic, undecylenic, nervonic, ricinoleic

Table 2. Utilization of unsaturated fatty acids and lecithin by human and bovine lipophilic corynebacteria

Compound $\quad \overbrace{\begin{array}{c}\text { Weak } \\ \text { positive }\end{array} \text { Negative }}^{\text {Response of } 39 \text { human strains }} \overbrace{\begin{array}{c}\text { Weak } \\ \text { Positive }\end{array} \text { positive Negative }}^{\begin{array}{c}\text { Response of eight } \\ \text { Corynebactrium bovis strains } \dagger\end{array}}$

fatty acids

cis oleic $\mathrm{C}_{18: 1}$

cis linoleic $\mathrm{C}_{18: 2}$

cis linolenic $\mathrm{C}_{18: 3}$

iso oleic $\mathrm{C}_{18: 1}$

(vaccenic)

trans oleic $\mathrm{C}_{18: 1}$

(elaidic)

Erucic $\mathrm{C}_{22: 1}$

Palmitoleic $\mathrm{C}_{\text {16:1 }}$

Lecithin

Positive

Negative

$\begin{array}{rrr}32 * & 5 & 2 \\ 7 & 17 & 15 \\ 0 & 3 & 36 \\ 25 & 13 & 1 \\ 34 & 3 & 2 \\ & & \\ 27 & 12 & 0 \\ 8 & 9 & 22 \\ 29 & 8 & 2\end{array}$

* Number of strains growing of total tested.

$\dagger$ American Type Culture Collection, ATCC 7715; National Institute for Research in Dairying, NIRD $37,65,66,112,126,128$.

Table 3. Inhibitory effect of certain fatty acids on the growth of human lipophilic corynebacteria

Tween 80 used at a concentration of $0.05 \%$ and fatty acids at $\mathrm{IO}^{-4} \mathrm{M}$.

Supplements added to basal medium

No. of strains
tested
36
36
29
16
16
24

No. of strains

Tween $80+$ linoleic acid

Tween $80+$ linolenic acid

Tween $80+$ ricinoleic acid

Oleic acid + linoleic acid

Oleic acid + linolenic acid

Oleic acid + ricinoleic acid

inhibited

6
5
2
0
0
I




\section{DISCUSSION}

The 39 human cutaneous lipophilic corynebacterial (LC) strains examined represented groups II to VII of the scheme proposed by Smith (1969a). There were no observed relationships between the fatty requirements of the strains and their tentative grouping, which were based on fermentation reactions. Considering the array of compounds tested, the 39 strains fall into a relatively narrow range in terms of the fatty acids required to initiate growth. Long chain fatty acids, particularly $\mathbf{C}_{\mathbf{1 8}}$ oleic acids, are essential. Trans, cis or iso oleic acid supported growth but ricinoleic acid did not. This may reflect the rôle of specific configurational changes in fatty acid utilization. Erucic acid $\mathrm{C}_{22: 1}$ supported growth but nervonic acid $\mathrm{C}_{24: 1}$ was not effective. Increased desaturation, as in linoleic and linolenic acids, resulted in decreased utilization by the majority of the human strains but few of the strains were inhibited. In the presence of oleic acid, compounds such as acetic, lactic and propionic acid did not enhance growth but longer chain acids such as palmitic acid did enhance growth. Many Grampositive bacteria are inhibited by the cis form of linoleic or linolenic acid (O'Leary, 1962). Pillsbury \& Rebell (1952) observed that cutaneous diphtheroids were inhibited by some fatty acids but in their tests, these authors used paper discs soaked with $10 \%$ solutions of the fatty acids; such a concentration is not physiological.

The samples of vaccenic, elaidic and erucic acids used here were only $95 \%$ pure but at the concentration used, impurities in the form of other fatty acids would not have exceeded $\mathrm{I} \cdot 8 \mu \mathrm{g} . / \mathrm{ml}$; ; this concentration, oleic acid alone did not support growth of the LC strains. The lipid-deficient basal broth may have been depleted of other metabolites which limited growth but with this medium it was shown that certain fatty acids were needed to initiate growth.

Some investigators recognize that various human LC stains resemble Corynebacterium bovis (Marples, 1969). Characterizations of human and bovine LC bacteria have shown numerous biochemical, physiological and nutritional differences between the two groups (Smith, I969a; Jayne-Williams \& Skerman, 1966). Skerman \& JayneWilliams (I966) reported that vaccenic, erucic, palmitoleic, ricinoleic and oleic acids supported the growth of $C$. bovis to various degrees. The failure of six of the eight $C$. bovis strains to respond in a way similar to the human LC stains may provide further evidence to distinguish between LC strains of human and animal origin.

The rôle of fatty acid utilization in the ecology of human cutaneous bacteria is a perplexing problem. Sarkany \& Gaylarde (1968) showed the localization of sebum to have characteristic patterns on skin. This could limit the distribution on skin of obligate lipophilic diphtheroids. Moreover, freshly collected sebum from intact sebaceous glands does not contain free fatty acids (Kellum, 1967) although some lipolytic strains of Corynebacterium acnes living in the pilosebaceous follicles or ducts liberate the fatty acids found in sebum (Reisner, Silver, Puhvel \& Sternberg, I968). The LC organisms are highly esterolytic but do not appear to contain true extracellular lipases (Smith, 1969a). In this respect, they appear to be similar to leptospires that are lipase-negative and require long-chain fatty acids (Johnson \& Harris, I968). JayneWilliams \& Skerman (1966) reported $C$. bovis strains to be lipolytic. More detailed studies of lipophilic-lipolytic relationships among the Corynebacteria are required. 


\section{REFERENCES}

Jayne-Williams, D. J. \& Skerman, T. M. (1966). Comparative studies on coryneform bacteria from milk and dairy sources. Journal of Applied Bacteriology 29, 72.

Johnson, R. C. \& Harris, V. G. (I968). Purine analogue and lipase activity of leptospires. Applied Microbiology 16, 1584.

Kellum, R. E. (1967). Human sebaceous gland lipids. Archives of Dermatology and Syphilology 95 , 218.

O'Leary, W. M. (1962). The fatty acids of bacteria. Bacteriological Reviews 26, 421.

MARPLes, R. R. (1969). Diphtheroids of normal human skin. British Journal of Dermatology 81, sup. I, 47 .

Pillsbury, D. M. \& Rebell, G. (1952). The bacterial flora of the skin. Factors influencing the growth of resident and transient organisms. Journal of Investigative Dermatology 18, 173.

Pollock, M. R., Wainwright, S. D. \& Mansion, E. D. (I949). The presence of oleic acid requiring diphtheroids on human skin. Journal of Pathology and Bacteriology 6r, 274.

Reisner, R. M., Silver, D. Z., Puhvel, M. \& Sternberg, T. H. (I968). Lipolytic activity of Corynebacterium acnes. Journal of Investigative Dermatology $\mathbf{5} \mathbf{I}, 190$.

SARKANY, I. \& GaYlarde, P. (1968). A method for demonstration of the distribution of sebum on the skin surface. British Journal of Dermatology 80, 744.

Skerman, T. M. \& JAYNE-Williams, D. J. (I966). Nutrition of coryneform bacteria from milk and dairy sources. Journal of Applied Bacteriology 29, I67.

SMITH, R. F. (1969a). Characterization of human cutaneous lipophilic diphtheroids. Journal of General Microbiology 55, 433 .

SMITH, R. F. (I969b). Medium to study the ecology of human cutaneous diphtheroids. Journal of General Microbiology 57, 4II.

Stinson, M. W. \& Solotorovsky, M. (1969). Breakdown and utilization of Tween 80 by Mycobacterium avium in synthetic medium. Bacteriological Proceedings p. 83.

WEARY, P. E. (1968). Pityropsorum ovale. Observations on some aspects of host-parasite interrelationships. Archives of Dermatology and Syphilology 98, 408. 